Case Report

\title{
An Unusual Pediatric Case of Allen Key Penetrating Trauma in Maxillofacial Region
}

\author{
Feride Fatma Görgülüi (iD, ${ }^{1}$ Orhan Görgülï,, ${ }^{2}$ Ayşe Selcan Koç, ${ }^{1}$ \\ and Fatma Yasemin Öksüzler ${ }^{1}$ \\ ${ }^{1}$ Radiology Department, University of Health Sciences Adana City Research and Training Hospital, Adana, Turkey \\ ${ }^{2}$ ENT Department, University of Health Sciences Adana City Research and Training Hospital, Adana, Turkey
}

Correspondence should be addressed to Feride Fatma Görgülü; drferide@yahoo.com

Received 24 November 2017; Accepted 14 March 2018; Published 23 April 2018

Academic Editor: Suayib Yalcin

Copyright (c) 2018 Feride Fatma Görgülü et al. This is an open access article distributed under the Creative Commons Attribution License, which permits unrestricted use, distribution, and reproduction in any medium, provided the original work is properly cited.

Paranasal sinus (PNS) foreign bodies are not common. They are usually due to penetrating trauma and iatrogenic events. On imaging, radiopaque foreign bodies can easily be detected by X-ray views of PNS. CT scan may be necessary to evaluate the exact location of foreign body in some cases. Foreign body in the PNS should be removed as early as possible. Approach and technique of its removal depend upon its size, shape, and location. Nasal endoscopic examination can be helpful for these cases. We present a pediatric girl case of penetrating FB injury inserted into the maxillary sinus towards nasopharynx in a suddenly braking car.

\section{Introduction}

Paranasal sinus foreign bodies (PNS FBs) are commonly secondary to gunshots and accidental sharp materials [13]. There was a reported case of an arrow, wooden foreign body, and wristwatch in the PNS. Foreign bodies in the PNS should be removed as early as possible. A retained foreign body can lead to sinusitis, cutaneous fistula, and foreign body granuloma formation [4-6].

Advanced imaging methods may be necessary in some cases whereas simpler radiological method can give us enough anatomical information about the FB in some other cases. We present a pediatric girl case of penetrating FB injury inserted into the maxillary sinus towards nasopharynx in a suddenly braking car. We would like to draw attention to choosing the radiological algorithm as first simpler to advanced ones in the examination of such patients besides being able to prevent cases like this trauma inside car.

\section{Case Report}

Nine-year-old pediatric girl patient came to the emergency department with the history of an Allen key injury on her left cheek (Figure 1). The family of the patient said that when the patient was traveling in the car, Allen key in her hand was inserted into her face because of the sudden brake.

On examination, an entry of foreign body was found on the upper left cheek. The tip of Allen key was seen at nasopharynx with diagnostic nasal endoscopy after the nasal decongestion. There were edema and tenderness over the left cheek. It revealed no hemorrhage of the turbinates or the nasal mucosa on anterior rhinoscopy. Firstly the lateral skull roentgenogram revealed Allen key in the left maxillary sinus to nasopharynx. The body of the Allen key has a very close relation to the floor of the orbit and the sphenoid sinus (Figure 2). This image was good enough for us, but the computed tomography (CT) was also used to make sure that the periorbital and skull base were damaged or not. CT of the maxillofacial region revealed no orbital and intracranial injury (Figure 3). The patient also had no neurological or vascular injury.

The patient was immediately operated on under general anesthesia. During the operation, the foreign body was removed after short-term cauterization with monopolar cautery due to its metallic nature by making a millimetric incision on the adjacent skin tissue to prevent skin damage 


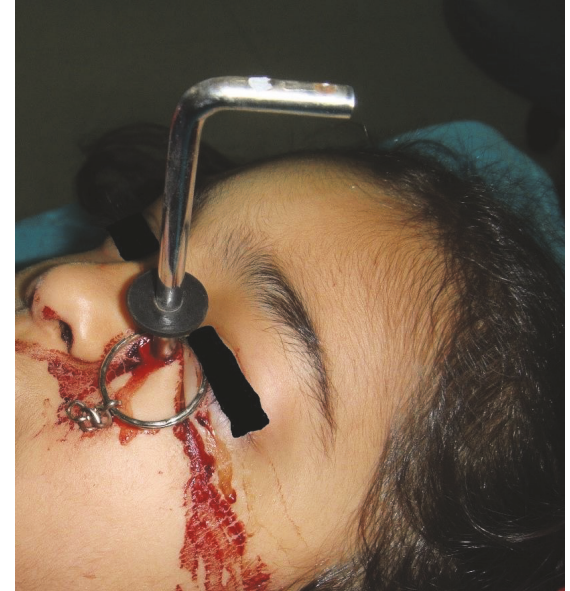

Figure 1: Photograph of the patient at the time of admission.

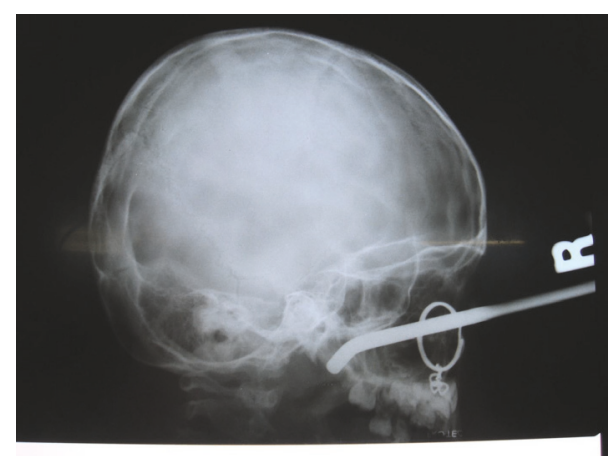

FIGURE 2: Preoperative X-ray of the skull lateral view, showing the Allen key in the left maxillary sinus to nasopharynx.

(Figure 4). This step was done to prevent active bleeding. The entry site and incision were sutured when no hemorrhage was seen after the foreign body was removed. The left nasal passages were again examined by nasal endoscopy and no abnormality was detected. Antibiotics were given for seven days to prevent possible infection. The patient was discharged on the same day of the operation.

At the 6-month follow-up, the patients' physical examination and radiological investigations were normal.

\section{Discussion}

Foreign bodies in the PNS are rare. It is difficult to predict the frequency of occurrence because of the small number of publications published in the literature. About $50 \%$ to $75 \%$ of all foreign bodies in the PNS are found in the maxillary sinuses [7-9]. Introduction of foreign bodies to the paranasal sinuses may occur through a variety of traumatic or iatrogenic events. Traumatic ones include pellets or bullets from gunshot injuries, wood, pieces of glass, and stones, while iatrogenic ones include teeth, dental cement, and pieces of broken forceps [10-12].

The diagnostic approach consists of a complete medical history, otolaryngological examination, and appropriate

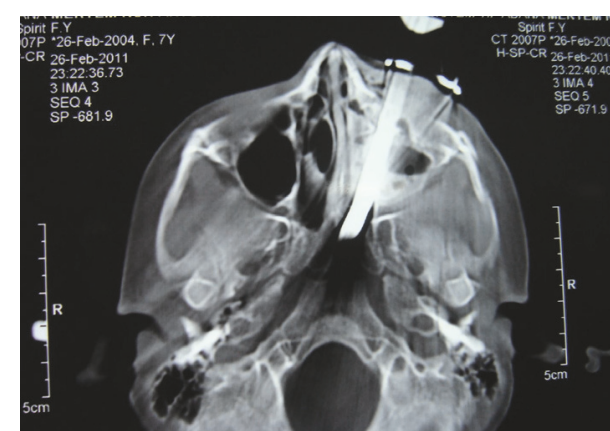

FIGURE 3: Foreign body visualised on CT at the time of admission.

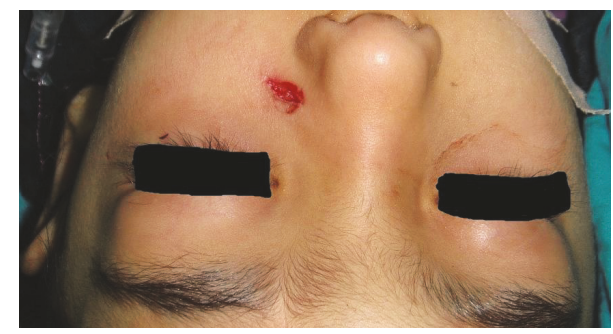

FIGURE 4: Photograph of the patient after removal.

radiographs. Patients coming in the emergency with blast injuries and penetrating trauma should undergo radiological investigation before operation. Waters and lateral skull views are useful imaging tools in diagnosing FB and CT scan can be usefully used to evaluate the presence and exact location of $\mathrm{FB}[8,13,14]$.

Foreign bodies in the PNS should be removed as early as possible. A retained foreign body can lead to sinusitis, cutaneous fistula, foreign body granuloma formation, and even malignant mucosal alteration. The general treatment guidelines for a penetrating maxillofacial injury are to decompress, debride, and avoid neurovascular injury and the subsequent complications. A multidisciplinary surgical intervention is always required. There are a variety of techniques for removing paranasal sinus FBs. Location of foreign body may require a variation in operative technique. The surgical approach must be chosen according to the mechanisms, patterns, and materials of the injury. The classic open surgical techniques are preferred in large FBs, whereas small foreign bodies can easily be removed endoscopically [15-19].

The surgical technique used in this case was unusual because the tip and body of the metallic FB were inside the face. Therefore there was a possible risk of significant bleeding, if we prefer to remove it simply by pulling it from skin. We removed it after short-term cauterization with monopolar cautery by making a millimetric incision on the adjacent skin tissue to prevent skin damage.

This case is unusual and interesting for three reasons; first, the foreign body itself, a whole Allen key, is the first in the literature to our knowledge; second, it also draws attention to the causing form of trauma, a girl in vehicle having Allen key in her hand that was preventable cause; third, our aim is 
to emphasise choosing simpler radiological methods rather than complicated ones.

\section{Conclusion}

Foreign bodies in the paranasal sinuses vary in their size and location. Approach and technique of their removal depend upon their size, shape, and location. In some cases simpler radiological view can give us enough information; in the other ones advanced imaging like MRI and angiography may be necessary.

\section{Conflicts of Interest}

The authors declare that there are no conflicts of interest regarding the publication of this paper.

\section{References}

[1] R. Blythe and M. Abbas-Ali, "An unusual case of maxillary sinus trauma," Journal of Surgical Case Reports, vol. 2012, no. 7, 4 pages, 2012.

[2] S. Misra, T. Anwar, and B. Basak, "An arrow penetrating at base of the skull successfully removed," Journal of the Indian Medical Association, vol. 108, no. 3, pp. 176-177, 2010.

[3] B. M. N. Saeed, "Traumatic foreign bodies in the paranasal sinuses," Jordan Medical Journal, vol. 47, no. 1, pp. 57-65, 2013.

[4] S. Harvinder, L. Jenny, and S. Gurdeep, "An arrow in the maxillary sinus," Medical Journal of Malaysia, vol. 63, no. 4, pp. 333-334, 2008.

[5] R. Sharma, R. Minhas, and N. Mohindroo, "An unusual foreign body in the paranasal sinuses," Indian Journal of Otolaryngology and Head \& Neck Surgery, vol. 60, no. 1, pp. 88-90, 2008.

[6] M. A. Mathews, B. A. Nair, S. Tandon et al., "Penetrating foreign body in the maxillary sinus and pterygopalatine fossa: report of a rare case," The Internet Journal of Head and Neck Surgery, vol. 4, 2010.

[7] D. Lee, M. Nash, J. Turk, and G. Har-El, "Low-velocity gunshot wounds to the paranasal sinuses," Otolaryngology-Head and Neck Surgery, vol. 116, no. 3, pp. 372-378, 1997.

[8] H. R. Krause, J. Rustemeyer, and R. R. Grunert, "Foreign body in paranasal sinuses," Mund-, Kiefer- und Gesichtschirurgie, vol. 6, no. 1, pp. 40-44, 2002.

[9] S. H. Batista, E. S. Soares, F. W. Costa et al., "Foreign body in the maxillary sinus. Considerations on maxillary sinus approaches wound closure," Revue De Stomatologie Et De Chirurgie MaxilloFaciale, vol. 112, pp. 316-318, 2011.

[10] K. M. Dodson, M. A. Bridges, and E. R. Reiter, "Endoscopic transnasal management of intracranial foreign bodies," JAMA Otolaryngology-Head \& Neck Surgery, vol. 130, no. 8, pp. 985988, 2004.

[11] D. Macan, T. Ćabov, P. Kobler, and Ž. Bumber, "Inflammatory reaction to foreign body (amalgam) in the maxillary sinus misdiagnosed as an ethmoid tumor," Dentomaxillofacial Radiology, vol. 35, no. 4, pp. 303-306, 2006.

[12] J. Yousefi, A. Mobasher Jannat, and R. Ajudani, "Penetrating nose and maxillary sinus injury with a metal part of a military gun," Journal of the Royal Army Medical Corps, vol. 162, no. 2, pp. 137-138, 2016.
[13] S. Sundereswaran, J. James, and S. Praveen, "Unusual foreign body in routine lateral cephalogram," Journal of Clinical and Diagnostic Research, vol. 9, no. 2, pp. ZD10-ZD11, 2015.

[14] C. Offiah and E. Hall, "Imaging assessment of penetrating injury of the neck and face," Insights into Imaging, vol. 3, no. 5, pp. 419$431,2012$.

[15] M. Akgüner, A. Atabey, and H. Top, "A case of self-inflicted intraorbital injury: Wooden foreign body introduced into the ethmoidal sinus," Annals of Plastic Surgery, vol. 41, no. 4, pp. 422-424, 1998.

[16] F. Pagella, E. Emanuelli, and P. Castelnuovo, "Endoscopic extraction of a metal foreign body from the maxillary sinus," The Laryngoscope, vol. 109, no. 2, pp. 339-342, 1999.

[17] N. Bhattacharyya and R. K. Wenokur, "Endoscopic Management of a Chronic Ethmoid and Sphenoid Sinus Foreign Body," Otolaryngology - Head and Neck Surgery, vol. 118, no. 5, pp. 687690, 2016.

[18] P. Y. Chen, S. F. Yao, A. X. Dai, H. J. Chen, and K. W. Wang, "A shocking craniofacial penetrating injury by a steel bar," Ulusal Travma ve Acil Cerrahi Dergisi, vol. 20, no. 5, pp. 382-384, 2014.

[19] S. Kazim, A.-U. Bhatti, and S. Godil, "Craniocerebral injury by penetration of a T-shaped metallic spanner: A rare presentation," Surgical Neurology International, vol. 4, no. 1, Article ID 106115, 2013. 


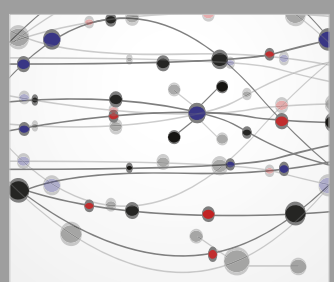

The Scientific World Journal
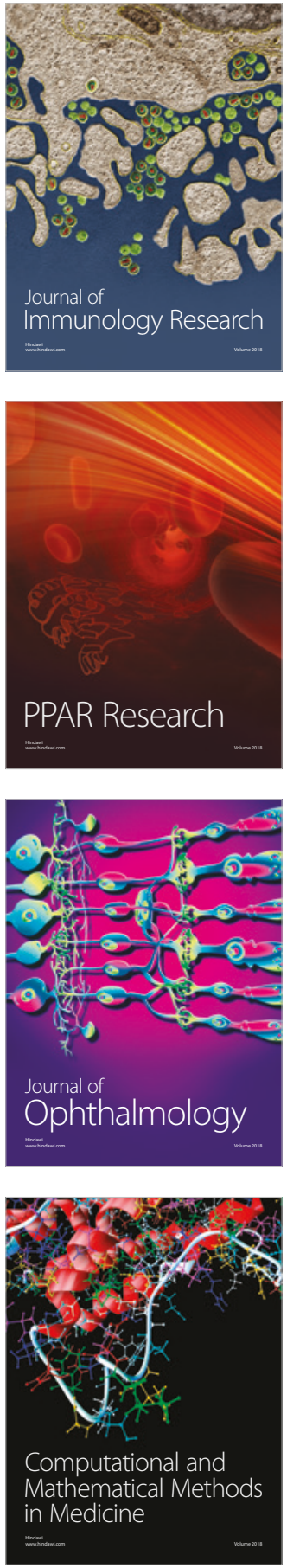

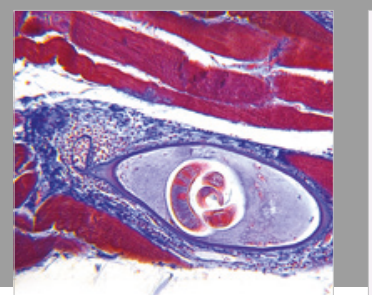

Gastroenterology Research and Practice

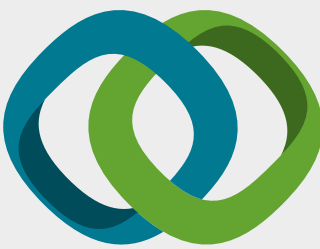

\section{Hindawi}

Submit your manuscripts at

www.hindawi.com
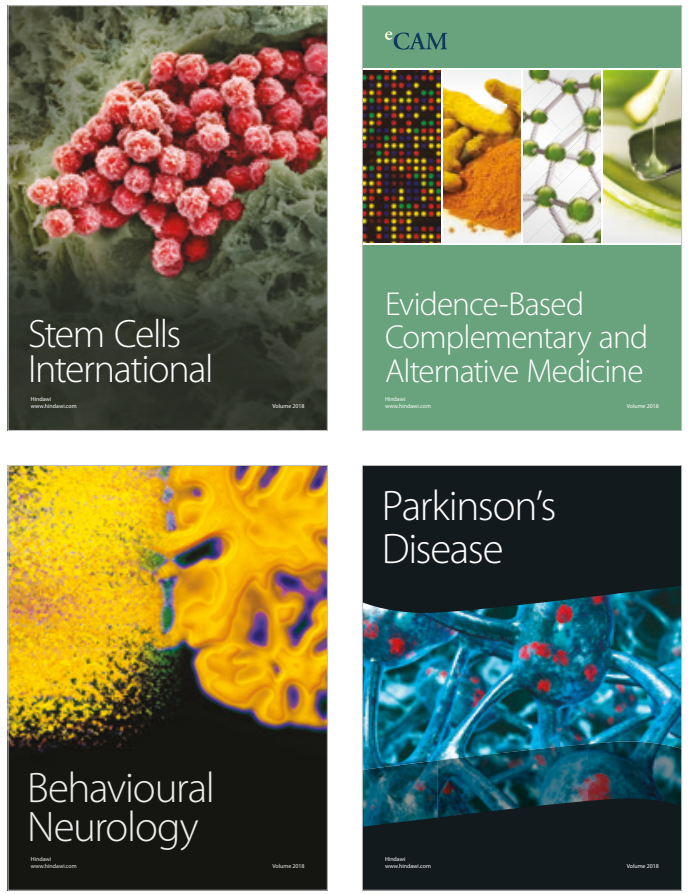

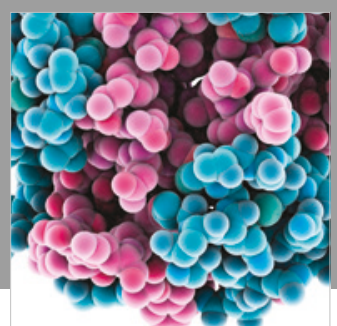

ournal of

Diabetes Research

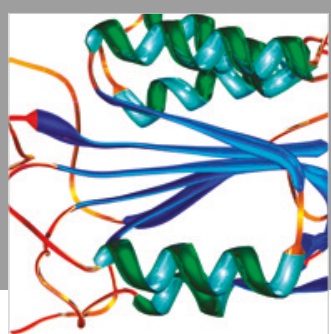

Disease Markers
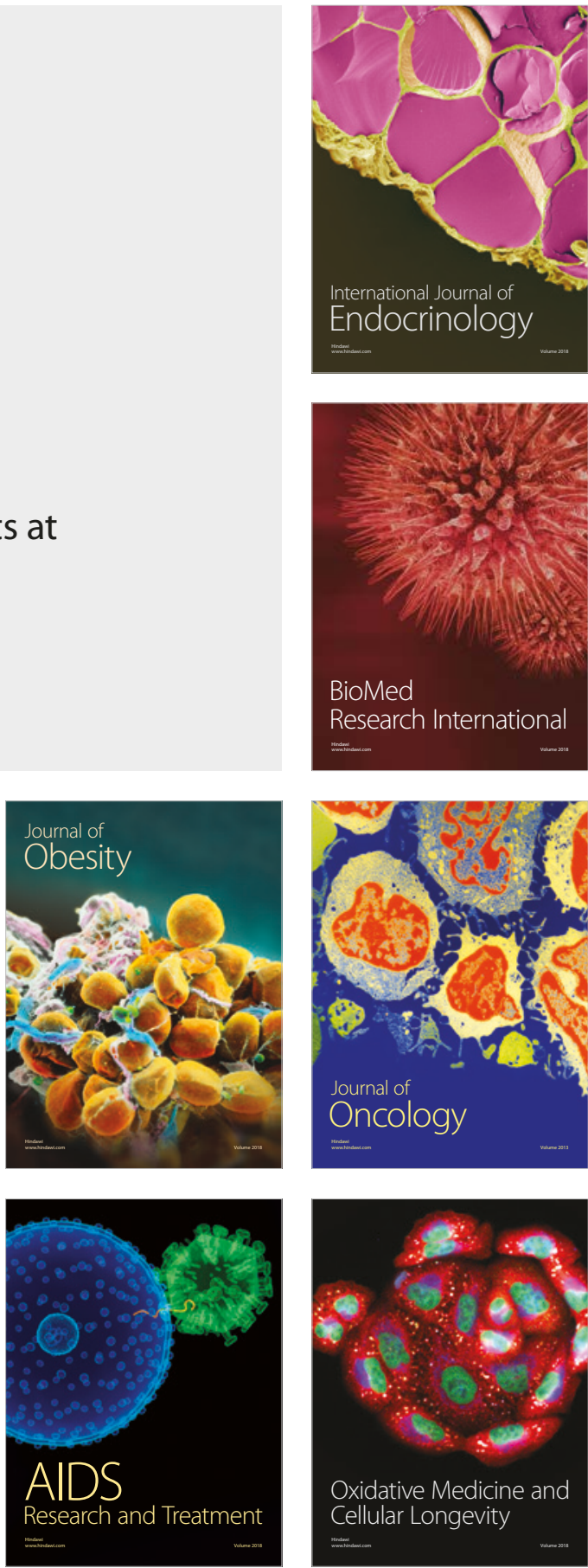